\title{
A “Non-Invasive” Technique for Qualifying the Reinforced Concrete Structure
}

\author{
Antonella Guida, Antonello Pagliuca, and Alessandro Tranquillino Minerva \\ Department of European and Mediterranean Cultures, School of Architecture, University of Basilicata, \\ Via Lazazzera, 75100 Matera, Italy \\ Correspondence should be addressed to Antonella Guida, arch.antonellaguida@gmail.com
}

Received 21 March 2012; Revised 4 June 2012; Accepted 30 June 2012

Academic Editor: Raffaele Solimene

Copyright ( 92012 Antonella Guida et al. This is an open access article distributed under the Creative Commons Attribution License, which permits unrestricted use, distribution, and reproduction in any medium, provided the original work is properly cited.

\begin{abstract}
In recent years, a lot of studies on built heritage emphasize the need to use appropriate techniques to evaluate the current condition of the structure before designing an intervention. The research focuses on the restoration of reinforced concrete buildings that begin to show signs of decay and deterioration. To verify the state of a building, it's possible to use the "destructive" methods (that require a local removal of material) and "nondestructive" tests. The combined results from different "nondestructive" tests are very interesting instruments to assess the concrete strength. This methodological approach can help to reduce the possible errors when using the sclerometer and ultrasonic tests separately; in this way, the combined method called "SonReb" (SONic + REBound) was developed. This paper would highlight the importance with respect to cultural heritage buildings and on the studied structure and contribute to developed engineering strategies to maintenance and restoration. The above-defined methodology has been tested on a postwar building which is located in Gravina in Puglia (Italy): the "Centrone" theatre; it was built using a mixed structure, that is, reinforced concrete and bearing masonry built of local stone. The building was used until the 1990s and now is abandoned. The analysis and qualification of the masonry structures of built heritage show how this approach is useful for classifying the pathological events on each building and to implement the innovative solutions to improve the durability of a restoration intervention.
\end{abstract}

\section{Introduction}

In the last decades, the architectural heritage of the modern movement seems to be more at risk than during any other period. This built inheritance embodies the dynamic spirit of the industrial age. At the end of the 1980s, many modern masterpieces had already been demolished or changed beyond recognition. This was mainly due to the fact that many were not considered to be elements of heritage that their original functions have substantially changed and that their technological innovations have not always endured long-term stresses.

A thorough research of built heritage enables understanding of the evolution of design philosophies and underlying cultural meanings and messages, artistic and functional qualities, and engineering achievements. A detailed knowledge of building materials, construction techniques, environmental services, external impacts, and internal impacts of use or disuse assist in identifying the problems affecting buildings and defining a methodological approach for interventions.

The research objective is to provide new qualitative information on the strength of reinforced concrete structures of a building by using innovative, non-invasive testing techniques. The case of study is the "Centrone" theatre in Gravina in Puglia (Italy).

The confluence of the local architectural styles (vernacular and academic) and the emerging aesthetic of reinforced concrete is explored to outline the context that influenced the building design.

The research methodology includes (a) the context in which the buildings were designed, (b) their history, (c) building technologies used, (d) non-invasive testing of the reinforced concrete structures, (e) the analysis of the test results, and (f) the conclusions. 
Testing methods could be "destructive", as they require a local removal of material, or "nondestructive", that is, they do not affect the structure. A sclerometer test, an ultrasonic test and their combined use, called SonReb (SONic + REBound), are "nondestructive" tests on reinforced concrete. The combined tests are a very useful method for assessing the concrete strength and to reduce the possibility of errors that can happen if the tests are not combined, as it has been noticed that the humidity content of a structural element can influence the sclerometer index and the ultrasound speed [1]. The combined method requires shorter time to obtain the results.

\section{A "Critical" Approach to Building Restoration}

The conservation intervention on a historic building, regardless of its architectural and/or artistic value, is generally more appropriate if information on its construction, evolution to date, materials, construction techniques, and structure is available. The conservation of built heritage highlights different issues such as the vast number of buildings needing attention and the urgency of cases that have to be resolved with limited economic resources and time.

To undertake a suitable intervention, three questions have to be answered: whether, where, and how to restore. The fourth question could be added, in which the economic aspect dominates: when to restore. To be able to respond adequately to these questions, it is necessary to proceed by developing specifications step by step, starting from a detailed knowledge of the entire building, the level and causes of degradation, and by finishing with the "operational" description of the proposed interventions.

Within the preliminary data acquisition phase, the direct survey of the building characteristics and condition and the mapping of any noticed changes are accompanied by the research on the project documentation and the events that have affected the structure during its construction and throughout the building life.

These approaches, often coupled with normative models which translate living conditions into objective parameters and standards, hinder the interventions such as a "simple" maintenance or a restoration or produce the result that is not logically related to the structural, typological, functional, and technological characteristics of the artefacts.

An "appropriate" restoration should plan the reuse of built heritage and aim to achieve building performance comparable to new buildings. The reuse interventions should be integrated with the conservation and not imposed.

The above methodology highlights how the approach could be helpful for the classification of pathological events within a building and for the application of innovative solutions to increase the durability of restoration interventions.

\section{The Investigative Techniques for Concrete}

The investigative techniques for concrete are also classified in two defined macrocategories ("destructive" and "nondestructive"). The former are based on the extraction of concrete samples to undertake compressive tests and represent the most reliable instrument for assessing the mechanical properties of concrete. The second investigative typology, the nondestructive tests, can be further subdivided in: really "nondestructive" investigations and "partially destructive" investigations. The latter include (a) the penetration test with a Windsor gun (ASTM C 83) which enables the identification of compressive resistance of concrete by measuring the depth of penetration of the special metal pins projected with a Windsor gun into concrete, (b) the extraction test (pullout) (UNI 10157:1992-ASTM C 900-06) which enables the assessment of compressive resistance of concrete by measuring the force used by a hydraulic jack for extracting a special plug inserted into concrete.

The really nondestructive investigations include, among others, (a) endoscopy that enables a direct observation of form and appearance of an investigated object, (b) thermography that assists in recognising potential structural anomalies by using the capacity of materials to transfer heat, (c) magnetometry which enables localising metal bars in reinforced concrete, (d) the Ground Penetrating Radar (GPR), especially with regard the investigation of the internal reinforcement bars, (e) measurement of the electric potential of concrete which enables defining the level of corrosion of metal reinforcements in concrete, (f) ultrasound investigation that allows qualitative assessment of the concrete resistance by using the capacity of the concrete components to transfer ultrasound waves, (g) sclerometric tests that assess the concrete resistance by reading the bounce results, and finally, (h) so-called "SonReb" (SONic + REBound) that enables assessing the concrete resistance by combining the speed of ultrasound waves and the index of surface bounce through a synergic use of the two previous investigations.

\subsection{The "SonReb" Method for Determining the Resistance of} Concrete. The SonReb method, as mentioned above, allows a qualitative determination of the concrete resistance [2] through the cross-examination of the values of the speed of ultrasound waves and the values of sclerometric bounce. This investigation method is standardised by RILEM Recommendations [3] 43 CND-EN 13791:2007, the EC regulation 12010 UNI EN 12504-2: 2001, ASTM C597, UNI EN 12504$4: 2005$, the Test Report CUR 69, the standards UNI 7997, UNI 9524 and UNI 83308.

This method is used for assessing the resistance of concrete, enabling the elimination of errors, at least partly, that appear when the two investigation methods are separately applied. This method, in fact, allows reducing the errors made when the sclerometric and ultrasound tests are undertaken separately [4].

It has been noticed, for example, that the humidity content leads to an underestimation of the sclerometric index and in an inversely proportional way leads to an overestimation of the ultrasound speed; similarly, the sclerometric index rises in a directly proportional way to the increase of the age of concrete [1], while the ultrasound speed decreases in an inversely proportional way to it [5].

In fact, the risk that can come up in the separate use of sclerometric and/or ultrasound tests is related, for example, 


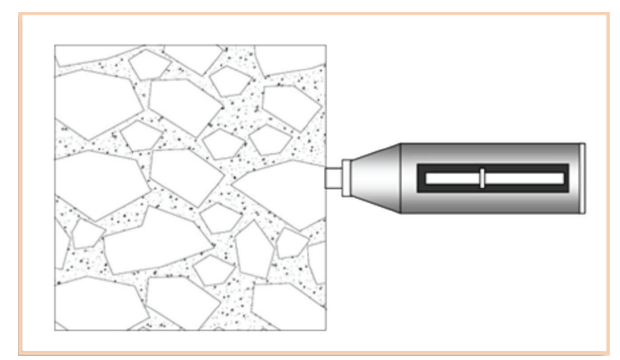

FIGURE 1: Sclerometer beats against a large piece of aggregate.

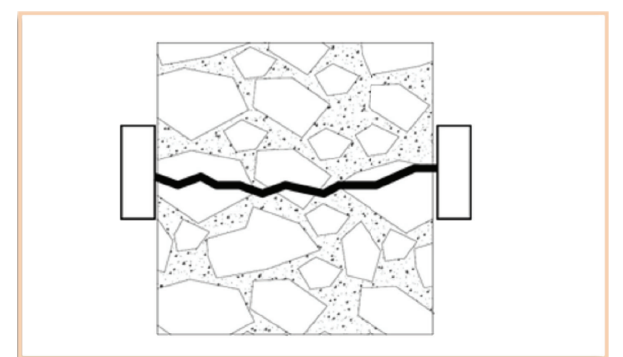

FIGURE 2: Ultrasound equipment: the impulse transmission.

to the chance nature of the position of aggregates in relation to the external surface of an investigated component; the sclerometer can easily beat against a large piece of aggregate, probably obtaining a higher value of the bounce index $S$ in relation to the one returned when the blow is executed on a homogenous concrete surface (Figure 1).

Again as an example, the ultrasound test (Figure 2) can equally be affected by the chance nature of the disposition of aggregates in a cement mix when the gaps between the aggregates are arranged in the way that induces the rise of the void index; in this case, the speed of wave spread $V$ decreases in relation to the value that would be obtained when the wave spreads through an area of "homogenous" concrete.

The application of the "SonReb" method asks for the appraisal [6] of local values of the ultrasound speed $V$ and of the bounce index $S$ from which it is possible to obtain the resistance of concrete $R_{c}$ through expressions such as:

$$
R_{c, \text { sonReb }}=a \cdot S^{b} \cdot V^{c} .
$$

In the scientific literature this formula has assumed different forms, each one expressing the experiments undertaken directly on site or in a laboratory on standardised samples.

For example:

(i) Gasparik [7] (1992)

$$
R_{c, 2}=8,06 \cdot 10^{-8} \cdot S^{1,246} \cdot V^{1,85},
$$

(ii) RILEM (1993) [2], NDT 4

$$
R_{c, 1}=9,27 \cdot 10^{-11} \cdot S^{1,4} \cdot V^{2,6}
$$

(iii) Di Leo and Pascale [6] (1994)

$$
R_{c, 3}=1,2 \cdot 10^{-9} \cdot S^{1,058} \cdot V^{2,446},
$$

in which:

(i) $R_{c}$ is the resistance of a cube under compression $\left[\mathrm{N} / \mathrm{mm}^{2}\right]$;

(ii) $S$ is the sclerometric index;

(iii) $V$ is the ultrasound speed $[\mathrm{m} / \mathrm{s}]$.

The formula (c) depicts the correlation curve applied in the investigated case studies. In (c) $R_{c}$ is expressed in $\mathrm{MPa}$ and the ultrasound speed in $\mathrm{m} / \mathrm{s}$; this relationship is related to a standard concrete whose properties are described in RILEM 43-CND. When a different type of concrete is employed the following relationship is applicable [8]:

$$
R_{\text {sonReb }}=R_{\text {sonReb }}^{\prime} \cdot\left(C_{c} \cdot C_{d} \cdot C_{a} \cdot C_{f} \cdot C_{P} \cdot C_{m}\right),
$$

where $R_{\text {sonReb }}^{\prime}$ is the value obtained from (1), while $C_{c}$ (cement type), $C_{d}$ (cement content), $C_{a}$ (aggregate types), $C_{f}$ (proportions of fines), $C_{p}$ (maximum aggregate size), and $C_{m}$ (errors) are coefficients of influence that permit the extension of (b) to the cases of a nonstandard concrete (as defined above).

Hence, if the concrete has the same characteristics as the one whose experimental curves are available, the graph directly provides the estimated resistance of concrete. Otherwise, as usually happens, when the concrete has a different composition from the one presented by the curve, the corrective coefficients, that take into account the type of cement and the related dosage, the nature and dimensions of aggregates, and the potential additives need to be applied to obtain an approximately true estimate of the concrete resistance [1].

It is evident that the above equations could not have universal validity except for the fact that the values of $S$ and $V$ depend on the characteristics of concrete, even when the specific indications on the limitations of applicability are missing.

However, a qualitative appraisal of the resistance of concrete can be made even by using the graphs (Figure 3 ) which show a series of isoresistance curves in the plane $V$ $S$ (obtained from the above analytic expressions) and which refer to the tests undertaken on standardised samples in a laboratory.

Although it appears absolutely necessary to analyse a wider range of cases, the above methodological investigation approach, based on the comparative analysis of the two described test campaigns, suggests several important considerations regarding the modality of investigation and the interpretation of results, demonstrating the need for establishing general investigation criteria: more than defining in a strict manner the number of tests that should be undertaken, it would be necessary to preset the level of significance to be achieved.

\section{The Case of Study: "Centrone" Theatre in Gravina in Puglia (Italy)}

The above-defined methodology has been tested on a postwar building which is located in Gravina in Puglia 


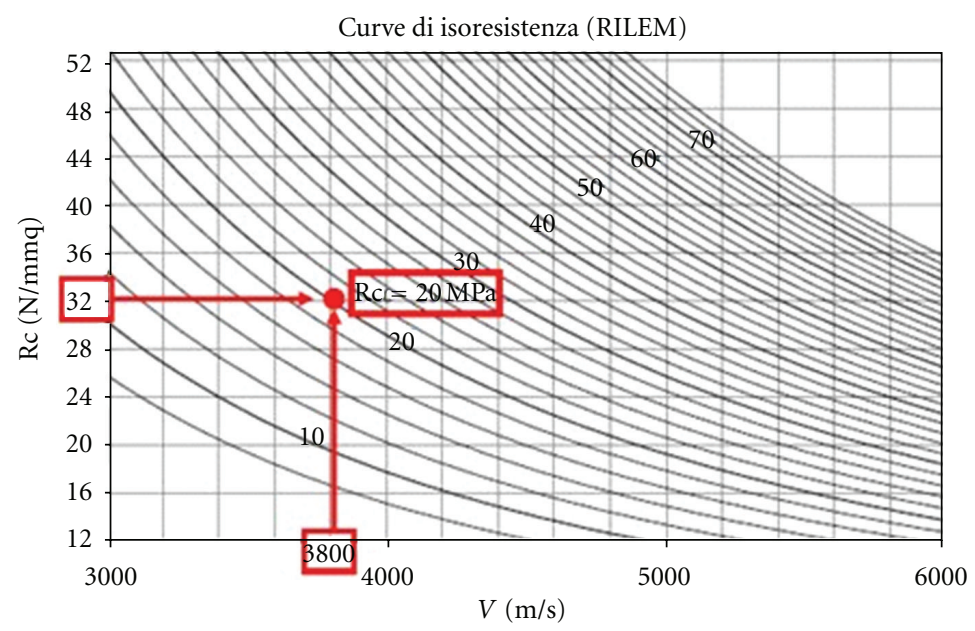

FIgURE 3: Example of isoresistance curves-RILEM NDT 4 recommendations.

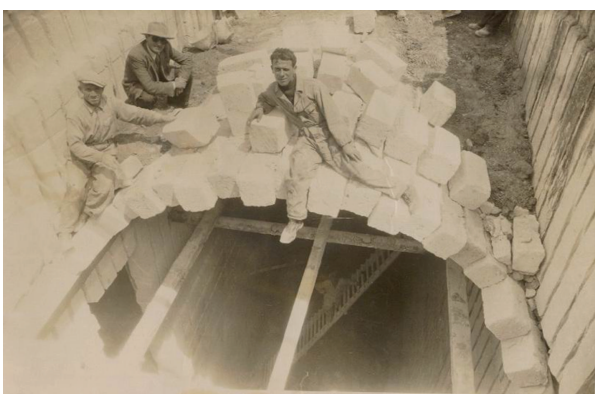

FIGURE 4: Historical image of vault construction.

(Italy): the "Centrone" theatre; it was built using a mixed structure, that is, reinforced concrete and bearing masonry built of local stone.

The building is not very old, but it is very interesting for the local civic history; in fact, it represents different values. Values that are set in different reels from the life of the building and revolved the surrounding environment in which it is inserted.

Historical value related to the historical development of the building and to the importance that it has in terms of expression of the local civic history; social value that is related to the social development of the city; cultural value that is related to the cultural growth of society; value of identity that has the building in terms of physics and metaphysics belong to a place then; but not least; the economic value related to the ability to reuse the building readapting it to new needs.

The building was built between 1946 and 1948; the foundations were built on pillars and vaults (Figure 4) and the pitched roof using iron trusses (Figure 5).

The building is a rectangular block (Figure 6), developed on three levels and covered using plaster and stone; the main façade is symmetrical, tripartite and follows the ground inclination; in the opposite side, the building shows two projecting polygonal elements realized using the local stone.

In the central part of the building; approximately $10 \mathrm{~m}$ wide; is located the entrance, highlighted by the massive

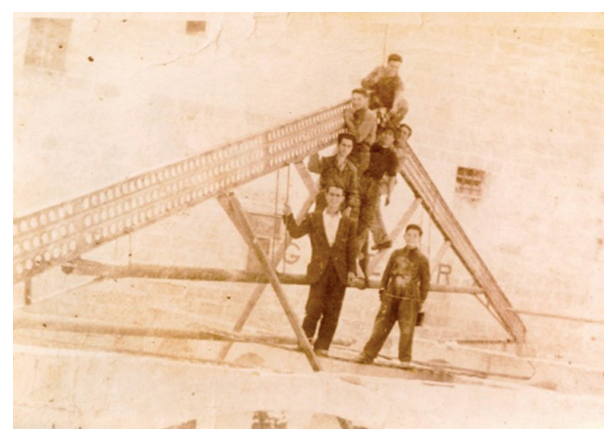

FIGURE 5: Historical image of iron trusses.

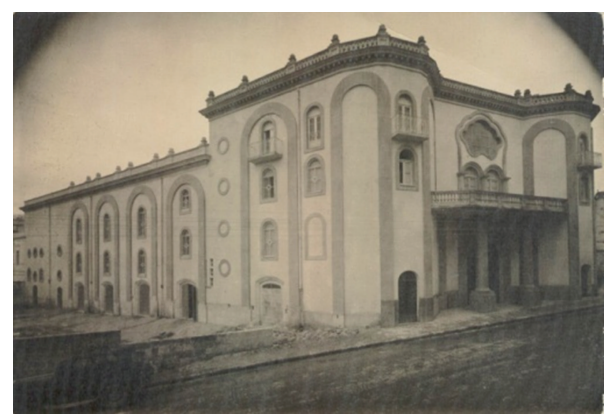

Figure 6: The "Centrone" theatre in Gravina in Puglia (Italy).

presence of two Doric columns that support a loggia. The building is organized in different spaces: the central part, where at ground floor there are the foyer entrance and ticket office, while on the first floor there is the Room "Italy" (Figure 7) that contains 110 seats; at second floor there are three flats for the managers of the theatre. The mail hall contains the stalls with 800 seats (a big space $32.5 \mathrm{~m}$ long and $21 \mathrm{~m}$ wide); it is partly covered by a balcony (at the first level) with 300 seats and a gallery on the second level with 300 seats.

The pathologies that interest the building are not particularly serious, since it does not affect in any way the 


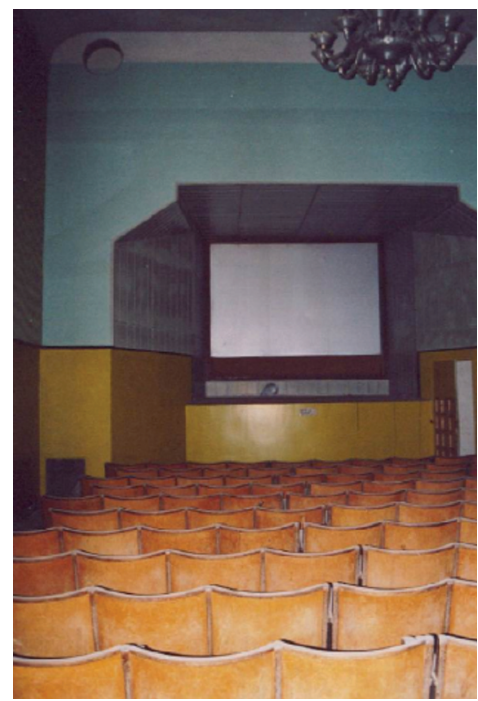

Figure 7: The room "Italy" on the first floor of the theatre.

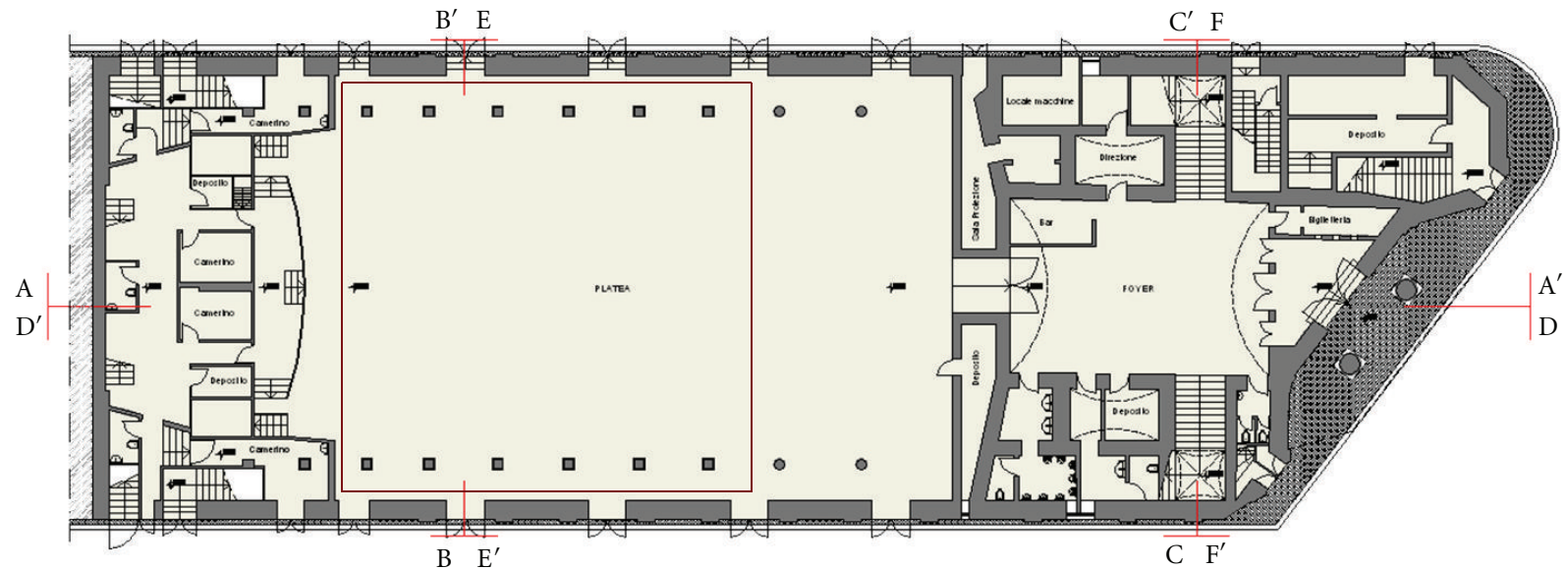

Figure 8: The specification of the location of the investigation points.

static system of structure and are easily solvable with noninvasive interventions. Inside are not detectable serious and manifest diseases, except for some water infiltrations from the roof that are determined as chromatic alterations and plaster detachment.

The condition of the pillars is generally acceptable, since they do not show obvious pathologies; however, you should note that some have a bad state of preservation of the plaster due to moisture that caused severe swelling, internal cavities and an evident surface lack of homogeneity.

4.1. On-Site Tests: Formulation. Essential elements for the organisation of an investigation campaign are the selection of the components that will be examined (which have to be representative of the whole structure), the investigation methodology that will be used, and the number and location of the investigation points; these requirements, in fact, are fundamental to guarantee a certain level of "reliability" of the obtained results and a "trustworthiness" with regard to the qualitative indications related to the characteristics of the material. In the case of the "Centrone" theatre, the identification of the components to be investigated was made by selecting the structural components which make the internal core of the load-bearing structure for two reasons: (1) because they enable to investigate a concrete in the state of "natural aging" (i.e., without considering the aggressive atmospheric agents which could have modified the condition of the material) and (2) for easiness of selecting the testing points. The structure of the whole building consists of a dual typology of structural components: those inserted in the external fabric (therefore difficult to investigate) and those located within the building.

The selection of the location of the investigation points (Figure 8) was random (with the aim to guarantee the representativeness of the investigation for the entire structure); in addition, "homogenous" areas [1] (which have the same characteristics) were noted and considered by making the obtained results "qualitative" and representative for all the 
TABLE 1: The value of rebound index.

\begin{tabular}{|c|c|c|c|c|c|c|c|c|c|c|c|c|}
\hline \multicolumn{2}{|c|}{ Structural element } & \multirow{2}{*}{\multicolumn{10}{|c|}{$\begin{array}{l}\text { Sclerometer value } \\
\text { Rebound value }\end{array}$}} & \multirow{3}{*}{$\begin{array}{c}\text { Average value } \\
28\end{array}$} \\
\hline \multirow{2}{*}{$\frac{\text { Element code }}{\text { P1 Dx T }}$} & \multirow{2}{*}{$\frac{\text { Size }[\mathrm{cm}]}{43 \times 43}$} & & & & & & & & & & & \\
\hline & & 27 & 28 & 26 & 29 & 25 & 25 & 31 & 26 & 28 & 28 & \\
\hline P2 Dx T & $43 \times 43$ & 34 & 38 & 36 & 42 & 40 & 36 & 37 & 38 & 41 & 38 & 38 \\
\hline P3 Dx T & $43 \times 43$ & 32 & 33 & 30 & 31 & 32 & 32 & 34 & 31 & 31 & 31 & 32 \\
\hline P4 Dx T & $43 \times 43$ & 35 & 33 & 34 & 30 & 36 & 34 & 34 & 34 & 36 & 38 & 38 \\
\hline P5 Dx T & $43 \times 43$ & 35 & 33 & 34 & 34 & 33 & 32 & 35 & 37 & 34 & 34 & 34 \\
\hline P6 Dx T & $43 \times 43$ & 35 & 37 & 38 & 38 & 36 & 34 & 35 & 38 & 36 & 36 & 36 \\
\hline P1 Sx T & $43 \times 43$ & 41 & 37 & 45 & 47 & 37 & 38 & 42 & 39 & 40 & 41 & 41 \\
\hline P2 Sx T & $43 \times 43$ & 43 & 42 & 42 & 40 & 44 & 42 & 46 & 44 & 44 & 40 & 43 \\
\hline P3 Sx T & $43 \times 43$ & 34 & 34 & 37 & 35 & 34 & 33 & 34 & 32 & 36 & 35 & 34 \\
\hline P4 Sx T & $43 \times 43$ & 32 & 28 & 32 & 32 & 34 & 34 & 34 & 34 & 30 & 32 & 32 \\
\hline P5 Sx T & $43 \times 43$ & 30 & 28 & 28 & 32 & 30 & 28 & 32 & 32 & 30 & 30 & 30 \\
\hline P6 Sx T & $43 \times 43$ & 34 & 35 & 34 & 36 & 35 & 37 & 38 & 35 & 38 & 35 & 36 \\
\hline P1 Dx I p & $43 \times 43$ & 23 & 21 & 21 & 22 & 22 & 25 & 25 & 26 & 24 & 22 & 23 \\
\hline P2 Dx I p & $43 \times 43$ & 33 & 32 & 33 & 32 & 30 & 30 & 30 & 33 & 31 & 30 & 31 \\
\hline P3 Dx I p & $43 \times 43$ & 37 & 40 & 38 & 41 & 43 & 38 & 38 & 38 & 41 & 38 & 39 \\
\hline P4 Dx I p & $43 \times 43$ & 38 & 38 & 38 & 38 & 37 & 39 & 37 & 38 & 39 & 39 & 38 \\
\hline P5 Dx I p & $43 \times 43$ & 37 & 39 & 35 & 38 & 37 & 38 & 38 & 40 & 38 & 40 & 38 \\
\hline P6 Dx I p & $43 \times 43$ & 36 & 38 & 37 & 37 & 38 & 38 & 37 & 38 & 36 & 38 & 37 \\
\hline P1 Sx I p & $43 \times 43$ & 26 & 22 & 24 & 22 & 24 & 20 & 23 & 22 & 22 & 22 & 23 \\
\hline P2 Sx I p & $43 \times 43$ & 40 & 41 & 37 & 40 & 40 & 39 & 38 & 38 & 37 & 38 & 39 \\
\hline P3 Sx I p & $43 \times 43$ & 40 & 37 & 35 & 37 & 36 & 35 & 35 & 35 & 35 & 35 & 36 \\
\hline P4 Sx I p & $43 \times 43$ & 35 & 35 & 35 & 37 & 35 & 37 & 36 & 38 & 36 & 38 & 36 \\
\hline P5 Sx I p & $43 \times 43$ & 40 & 40 & 37 & 35 & 36 & 35 & 38 & 36 & 36 & 36 & 37 \\
\hline P6 Sx I p & $43 \times 43$ & 36 & 38 & 36 & 38 & 38 & 38 & 40 & 40 & 38 & 38 & 38 \\
\hline
\end{tabular}

components that show the same characteristics; a further condition is that the selected surfaces do not show any obvious condition of degradation. The methodology used to undertake the tests was exclusively selected in terms of the possibility to undertake the tests. Regarding the sclerometric tests, they were undertaken by placing the instrument always orthogonally in relation to the investigated surface; the preference was given to the direct method of investigation. The considered reference (in terms of the number of tests with the aim to guarantee the reliability of the results) for the investigation campaign was taken from the framework of the Italian investigation procedures.

4.2. On-Site Tests: Analysis and Results. Sclerometer tests were carried out following the directions contained in UNI EN 12504-2 (2001). The instrument used is the concrete GEI model. On each pillar identified, it was carried out 10 measurements (Table 1) of the value of rebound (for each test, ten values were taken, from which the average value was considered as illustrative).

Three different areas were selected for each investigated component; at $0.70 \mathrm{~m}$, at $1.40 \mathrm{~m}$, and at $2.10 \mathrm{~m}$ from the floor. This selection, in fact, was motivated by the need to investigate the columns at the points of major stress (base and middle), having assumed (1) a uniform distribution of the loads and imposed loads (due to the homogenous structure) and (2) a homogenous condition of the loads (there are no visible cracks to induce any different considerations).

Ultrasonic tests were carried out according to the UNI EN 12504-4 (2005). Ultrasonic equipment used is the DSP model of Ultrasonic UTD 1004. On each pillar identified, it was carried out 10 measurements (Table 2) of the value of rebound (for each test ten values were taken, from which the average value was considered as illustrative).

These data were compared with the method "SonReb" (Tables 3, 4, 5, and 6) to improve-as said before-the qualitative interpretation of results; the analysis showed satisfactory results of the state of concrete condition, as well as the importance to compare the ultrasonic and sclerometer tests.

The obtained results show a significant homogeneity of values measured at each investigated level; more precisely, the sclerometric tests show an average value (of the ten measurements made at each point) of the bounce index uniformly distributed on the surfaces; whereas, the ultrasound tests returned average values of the ultrasound between a minimum value of $3050 \mathrm{~m} / \mathrm{s}^{2}$ and a maximum value of $3660 \mathrm{~m} / \mathrm{s}^{2}$. Comparing the results by using the method SonReb, the obtained values of the concrete resistance are from a minimum value of $18 \mathrm{MPa}$ to a maximum value of 
TABLE 2: The value of ultrasonic speed.

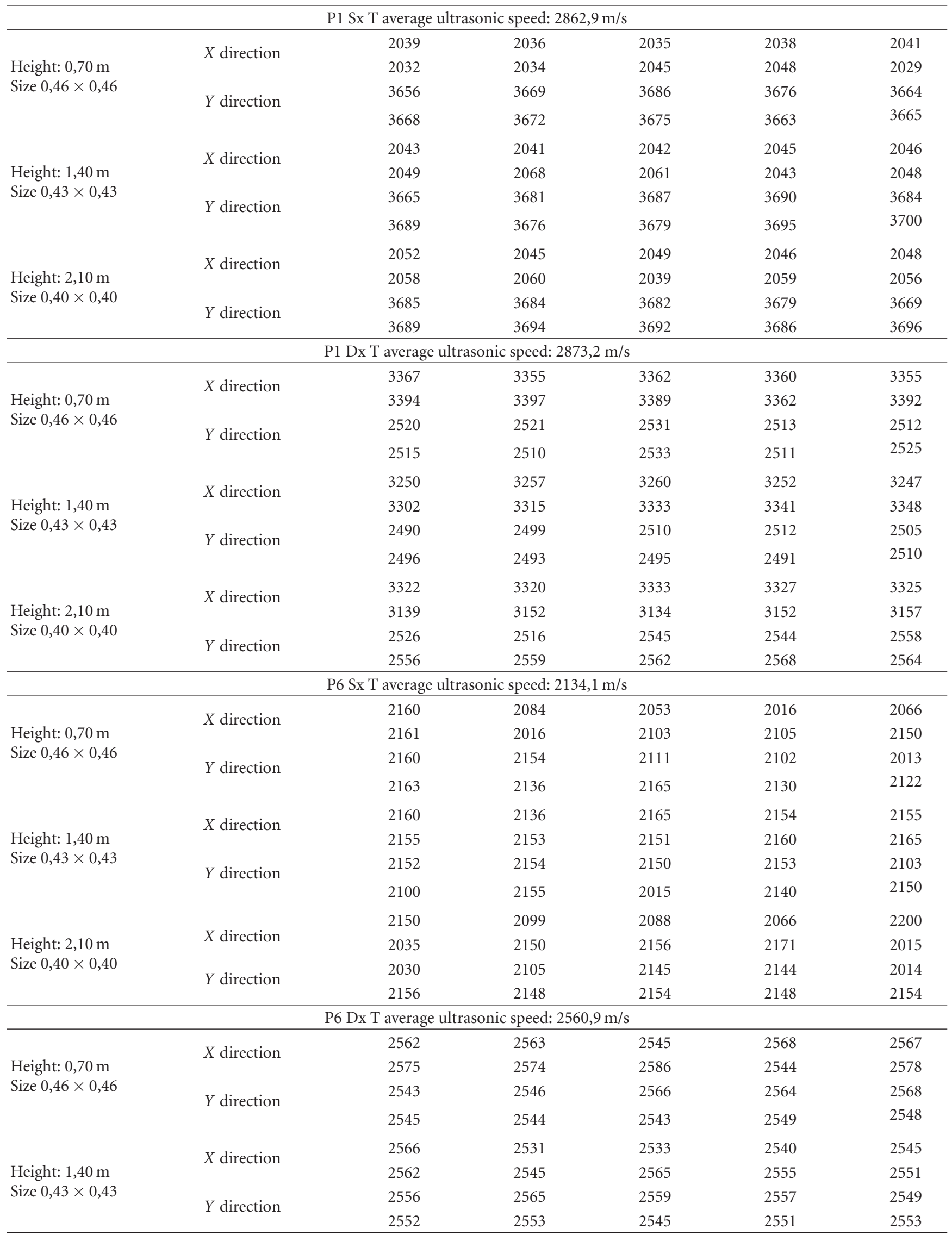


TABle 2: Continued.

\begin{tabular}{|c|c|c|c|c|c|c|}
\hline \multicolumn{7}{|c|}{ P6 Dx T average ultrasonic speed: $2560,9 \mathrm{~m} / \mathrm{s}$} \\
\hline \multirow{4}{*}{$\begin{array}{l}\text { Height: } 2,10 \mathrm{~m} \\
\text { Size } 0,40 \times 0,40\end{array}$} & \multirow{2}{*}{$X$ direction } & 2565 & 2596 & 2586 & 2567 & 2568 \\
\hline & & 2564 & 2572 & 2575 & 2574 & 2573 \\
\hline & \multirow{2}{*}{$Y$ direction } & 2564 & 2561 & 2563 & 2559 & 2568 \\
\hline & & 2567 & 2586 & 2584 & 2583 & 2569 \\
\hline \multicolumn{7}{|c|}{ P1 Sx I p average ultrasonic speed: $2582,0 \mathrm{~m} / \mathrm{s}$} \\
\hline \multirow{4}{*}{$\begin{array}{l}\text { Height: } 0,70 \mathrm{~m} \\
\text { Size } 0,46 \times 0,46\end{array}$} & \multirow{2}{*}{$X$ direction } & 1899 & 2130 & 2156 & 2102 & 2105 \\
\hline & & 2111 & 2182 & 2221 & 2310 & 2229 \\
\hline & \multirow{2}{*}{$Y$ direction } & 3200 & 3180 & 3196 & 3102 & 3104 \\
\hline & & 3016 & 3038 & 3086 & 3048 & 3062 \\
\hline \multirow{4}{*}{$\begin{array}{l}\text { Height: } 1,40 \mathrm{~m} \\
\text { Size } 0,43 \times 0,43\end{array}$} & \multirow{2}{*}{$X$ direction } & 1700 & 1750 & 1890 & 1806 & 1830 \\
\hline & & 1834 & 1905 & 1942 & 1972 & 1973 \\
\hline & \multirow{2}{*}{$Y$ direction } & 3109 & 3140 & 3149 & 3131 & 3158 \\
\hline & & 3160 & 3263 & 3264 & 3285 & 3189 \\
\hline \multirow{4}{*}{$\begin{array}{l}\text { Height: } 2,10 \mathrm{~m} \\
\text { Size } 0,40 \times 0,40\end{array}$} & \multirow{2}{*}{$X$ direction } & 2005 & 2103 & 1999 & 2150 & 2015 \\
\hline & & 2060 & 2160 & 2163 & 2184 & 2150 \\
\hline & \multirow{2}{*}{$Y$ direction } & 3005 & 3158 & 3139 & 3190 & 3100 \\
\hline & & 3089 & 3048 & 3069 & 3099 & 3105 \\
\hline \multicolumn{7}{|c|}{ P1 Dx I p average ultrasonic speed: $3006,5 \mathrm{~m} / \mathrm{s}$} \\
\hline \multirow{4}{*}{$\begin{array}{l}\text { Height: } 0,70 \mathrm{~m} \\
\text { Size } 0,46 \times 0,46\end{array}$} & \multirow{2}{*}{$X$ direction } & 3000 & 3002 & 3005 & 3019 & 2993 \\
\hline & & 3010 & 3015 & 3019 & 2921 & 2996 \\
\hline & \multirow{2}{*}{$Y$ direction } & 3012 & 3015 & 3018 & 3016 & 3018 \\
\hline & & 3001 & 3021 & 3029 & 3028 & 3020 \\
\hline \multirow{4}{*}{$\begin{array}{l}\text { Height: } 1,40 \mathrm{~m} \\
\text { Size } 0,43 \times 0,43\end{array}$} & \multirow{2}{*}{$X$ direction } & 3000 & 3052 & 3013 & 3002 & 3015 \\
\hline & & 2999 & 3050 & 3015 & 3016 & 3019 \\
\hline & \multirow{2}{*}{$Y$ direction } & 2996 & 3047 & 3036 & 3033 & 3031 \\
\hline & & 3002 & 3052 & 3035 & 3063 & 3043 \\
\hline \multirow{4}{*}{$\begin{array}{l}\text { Height: } 2,10 \mathrm{~m} \\
\text { Size } 0,40 \times 0,40\end{array}$} & \multirow{2}{*}{$X$ direction } & 2899 & 2915 & 3005 & 3001 & 3045 \\
\hline & & 3015 & 3062 & 3036 & 3035 & 3046 \\
\hline & \multirow{2}{*}{$Y$ direction } & 3051 & 3015 & 3063 & 3026 & 3033 \\
\hline & & 3018 & 3017 & 3021 & 3012 & 3026 \\
\hline
\end{tabular}

TABle 3: Left side pillar (Sx): Ground floor.

\begin{tabular}{lcc}
\hline Pillar & Average rebound value & Average ultrasonic speed \\
\hline P1 Sx T & 41 & 2862,9 \\
P2 Sx T & 43 & 3297,1 \\
P3 Sx T & 34 & 3345,3 \\
P4 Sx T & 32 & 3181,2 \\
P5 Sx T & 30 & 3192,1 \\
P6 Sx T & 36 & 2134,1 \\
\hline
\end{tabular}

$22 \mathrm{MPa}$, that take in a significant consideration the contribution of the covered concrete conditions.

\section{Conclusions}

The tests carried out are the basis of a diagnostic project that is possible to implement and monitor to guarantee
TABLE 4: Right side pillar (Dx): Ground floor.

\begin{tabular}{lcc}
\hline Pillar & Average rebound value & Average ultrasonic speed \\
\hline P1 Dx T & 28 & 2873,2 \\
P2 Dx T & 38 & 3103,6 \\
P3 Dx T & 32 & 3199,3 \\
P4 Dx T & 38 & 3313,2 \\
P5 Dx T & 34 & 3082,1 \\
P6 Dx T & 36 & 2560,9 \\
\hline
\end{tabular}

a deeper knowledge, with the goal of attaining a level of thorough understanding aimed at the "preservation and improvement" of a building.

The recovery project, that takes particular care in the methodological application of the diagnostic phase, cannot leave out of consideration the necessity of a careful and timely monitoring of building conditions. 
TABLe 5: Left side pillar (Sx): First floor.

\begin{tabular}{lcc}
\hline Pillar & Average rebound value & Average ultrasonic speed \\
\hline P1 Sx I p & 23 & 2582,0 \\
P2 Sx I p & 39 & 3476,1 \\
P3 Sx I p & 36 & 3358,2 \\
P4 Sx I p & 36 & 3319,6 \\
P5 Sx I p & 37 & 3450,3 \\
P6 Sx I p & 38 & 3346,1 \\
\hline
\end{tabular}

TABLE 6: Right side pillar (Dx): First floor.

\begin{tabular}{lcc}
\hline Pillar & Average rebound value & Average ultrasonic speed \\
\hline P1 Dx I p & 23 & 3006,5 \\
P2 Dx I p & 31 & 3210,2 \\
P3 Dx I p & 39 & 3120,9 \\
P4 Dx I p & 38 & 3204,6 \\
P5 Dx I p & 38 & 3098,1 \\
P6 Dx I p & 37 & 3301,5 \\
\hline
\end{tabular}

The carried out tests are the first and simplest analysis for a qualitative assessment; it is necessary to classify the structure regarding the following consolidation procedures. The recovery and conservation project, as well as an "indispensable" transformation of an old building, must be in that evaluations, of feasibility and suitability, both economic and practice which is the basis for a "suitable choice" of recovery intervention, that permit to annul the "cancellation" of the Built Heritage.

\section{References}

[1] A. Masi, La Stima della Resistenza del Calcestruzzo in situ Mediante Prove Distruttive e non Distruttive, Il Giornale delle Prove non Distruttive Monitoraggio Diagnostica no. 1, 2005.

[2] RILEM Draft Recommendation, 43-CND. Combined nondestructive testing of concrete. Draft recommendation for in situ concrete strength determination by combined nondestructive methods. Materials and Structures no. 26, 1993.

[3] RILEM (The International Union of Testing and Research Laboratories for Materials and Structures) is an organisation which enables exchanges through an international network of testing engineers, researchers, academics, educators and practitioners.

[4] F. Braga, M. Dolce, A. Masi, and D. Nigro, Valutazione delle Caratteristiche Meccaniche dei Calcestruzzi di Bassa Resistenza Mediante Prove non Distruttive, L'Industria Italiana del Cemento no. 3, 1992.

[5] P. Bocca and S. Cianfrone, "Le prove non distruttive sulle costruzioni: una metodologia combinata," L'Industria Italiana del Cemento no. 6, 1986.

[6] A. Di Leo and G. Pascale, Prove Non Distruttive Sulle Costruzioni in Cemento Armato, Convegno Sistema Qualità e Prove non Distruttive per l'affidabilità e la Sicurezza delle Strutture Civili, Bologna, Italy, 1994.

[7] J. Gasparik, Prove Non Distruttive Nell'edilizia, Università di Brescia, 1992.

[8] R. Giochetti and L. Lacquaniti, Controlli non Distruttivi su Impalcati da Ponte in Calcestruzzo Armato, Nota Tecnica 04, Università degli Studi di Ancona, Facoltà di Ingegneria, Istituto di Scienza e Tecnica delle Costruzioni, 1980. 

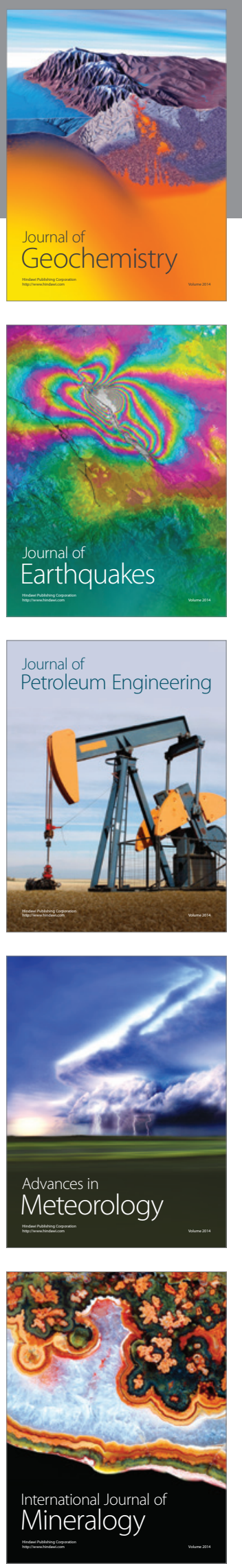
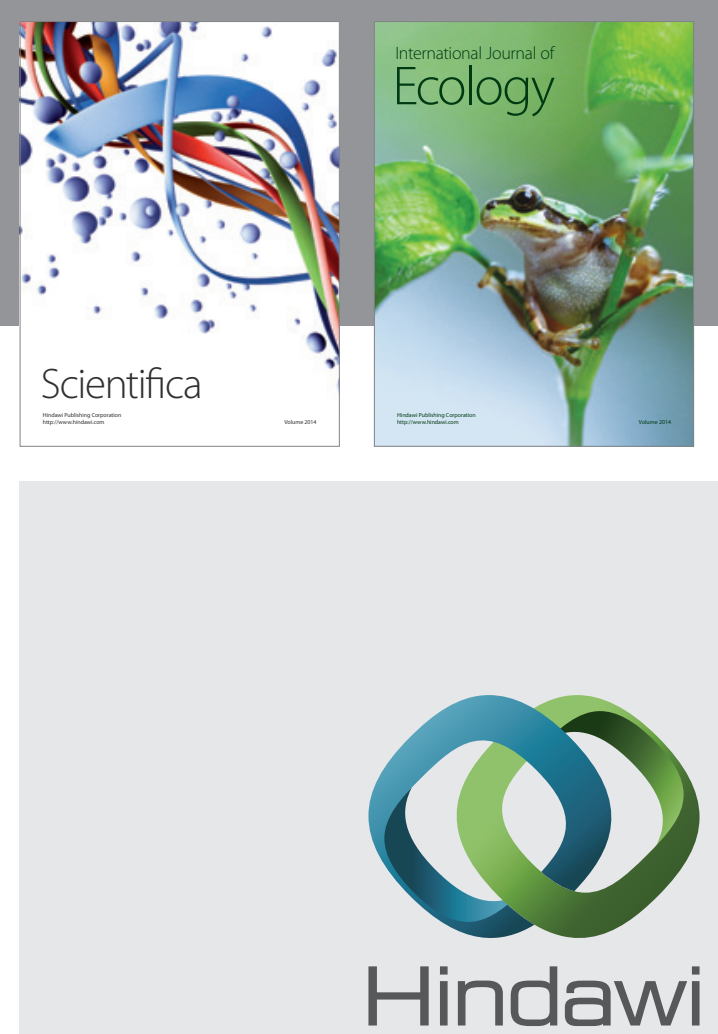

Submit your manuscripts at http://www.hindawi.com
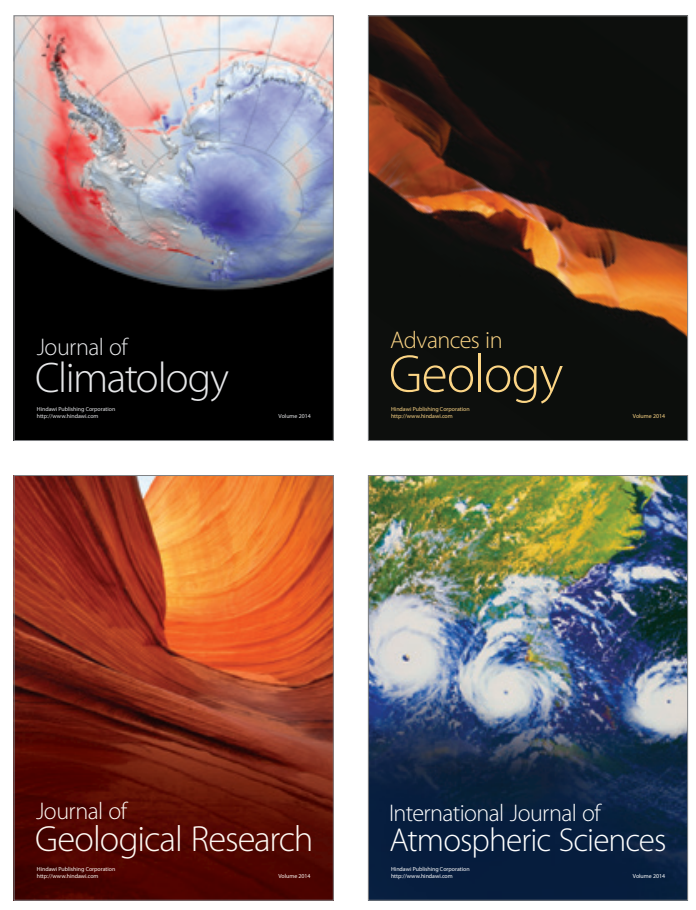
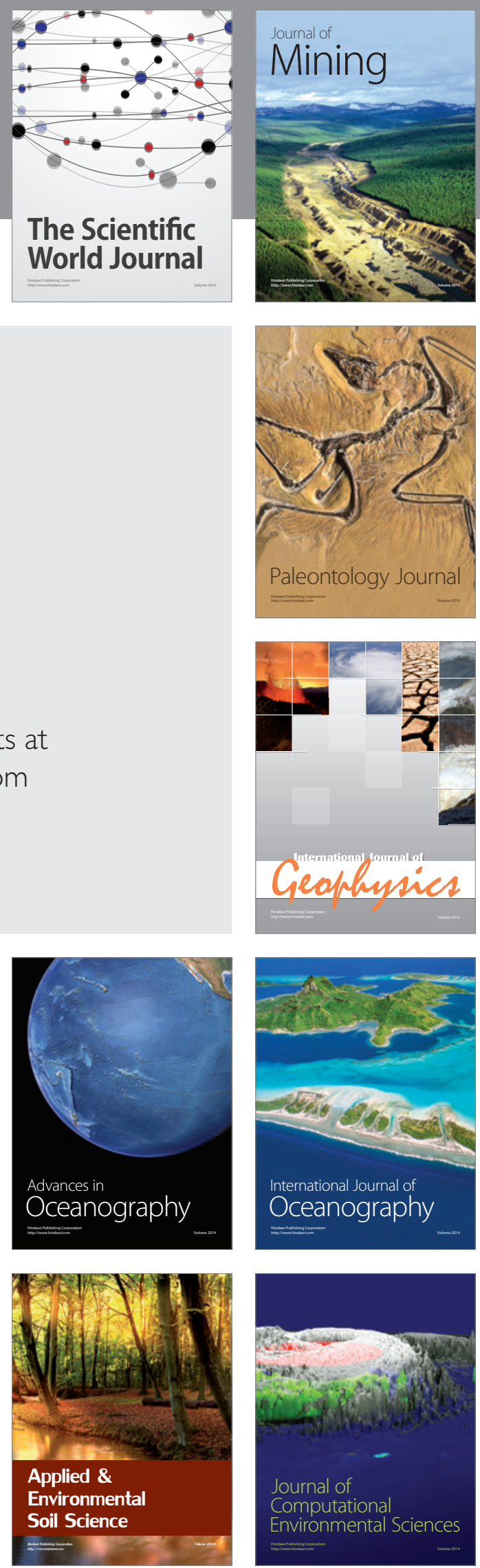